\title{
Checkmate to CHK1 in T-cell ALL?
}

\section{Leonor M. Sarmento and João T. Barata}

DNA replication ensures accurate duplication of the original genetic information present in a cell in order for it to be properly transmitted to daughter cells. However, replication can be perturbed, for instance in rapidly dividing cancer cells, in a process referred to as replication stress (RS). Checkpoint kinase 1 (CHK1) is an essential component of the ATR-dependent DNA damageresponse pathway that protect cells from RS by preventing replication fork collapse and activating homologous DNA repair. The ATR-CHK1 pathway is triggered upon exposure of single-stranded DNA that arises with the stalling of replication forks [1], and it is required to reset proper origin firing, and to promote fork stability and checkpoint activation, delaying mitosis until replication is completed and thereby avoiding mitotic catastrophe [2]. Whereas these functions point towards a tumor suppressor role for $\mathrm{CHK} 1$, mouse models modulating ATR-CHK1 expression and genetic evidence from human tumors suggest otherwise: Atr and Chkl knock-out models do not display higher tumor frequency; Chkl favors oncogeneinduced transformation in mice; $C H K 1$ is frequently overexpressed in human cancers, while loss-of-function mutations are rare [2, 3]. Moreover, CHK1 affords protection against DNA damaging agents, a fact that prompted the use of CHK1 inhibitors as chemosensitizers [4]. Similarly, tumors whose oncogenic profile fuels RS were proposed to become addicted to ATR-CHK1 response $[1,2]$. In our recent study published in Oncogene [5], we hypothesized that T-cell acute lymphoblastic leukemia (T-ALL), an aggressive hematological cancer arising from T-cell precursor clonal expansion, could be one of such tumors and showed that CHK1 plays a key role in T-ALL cell maintenance.

T-ALL cells tend to be highly proliferative due to a myriad of genetic lesions that culminate in cyclin-dependent kinase hyperactivation, and deregulated progression of S-phase that may impact on DNA replication $[5,6]$. We found that T-ALL cells overexpressed CHK 1 mRNA and protein as compared to normal hematopoietic progenitors. This was accompanied by aberrantly high CHK1 kinase activity, likely triggered by high basal levels of RS [5]. Experimental inactivation of CHK1, by a CHK1 selective inhibitor (PF-00477736) or by gene silencing, demonstrated that $\mathrm{CHK} 1$ is essential to control the accumulation of RS and to prevent apoptosis of T-ALL cells that appear to enter mitosis without having concluded DNA replication. Furthermore, accumulation of DNA damage in the context of CHK1 loss induced the activation of the ATM-CHK2 DNA double-strand break (DSB) response pathway, likely due to DSB formation upon the collapse of stalled replication forks. T-ALL apoptosis upon $\mathrm{CHK} 1$ inactivation was in the first instance dependent on ATM and caspase-3, since ATM inhibition prevented caspase- 3 cleavage and rescued T-ALL cell viability despite sustained elevated amounts of RS markers [5].

Following the demonstration that T-ALL cells were eliminated using a CHK1 small molecule inhibitor as single agent, we showed that this effect was leukemiaspecific, since normal T-cell progenitors were not sensitive to the low doses of PF-00477736 that killed primary T-ALL patient cells. Moreover, the in vitro anti-leukemia effect of PF-00477736 was not prevented by microenvironment pro-survival factors, and the potential clinical value of CHK1 inhibition was further demonstrated by the fact that PF-00477736 limited the growth of xenografted T-ALL tumors [5]. Interestingly, our preliminary analyses indicated that T-ALL cells expressing higher CHK1 levels appeared more sensitive to CHK1 pharmacological inhibition, suggesting that $\mathrm{CHK} 1$ expression could be a suitable drug response marker in T-ALL patients. As clinical trials against ATR-CHK1 pathway may be envisaged, this issue warrants extended T-ALL patient analysis.

T-ALL constitutes only a fraction of all ALL cases, but it associates with high-risk. Therapeutic options with less detrimental side-effects and/or effective upon relapse are most desired. Our findings defining CHK1 as a 'subverted' tumor suppressor that stands in T-ALL as a major guardian of leukemia cell survival, thereby formally acting as an oncogene, reinforce a new way of viewing the mechanisms of cancer progression [2] and may set the ground for anti-leukemia breakthrough approaches. In this context, it is important to understand the mechanisms of CHK1 upregulation in T-ALL. We thoroughly documented CHK1 transcript overexpression in primary T-ALL [5]. However, how this occurs remains undetermined. Maybe transcription factors known to activate $C H K 1$, such as E2F (downstream of G1/S-phase CDK activity) or MYC (downstream of NOTCH1), are involved in CHK1 overexpression in T-ALL. Or maybe as yet unidentified CHK1 regulatory elements are mutated or epigenetically altered. Curiously, in contrast to our findings, Chkl mRNA downregulation was documented in a murine 
T-ALL model [7]. A more integrative view of the role of CHK1 in T-ALL is therefore required. We believe CHK1 downregulation may occur at T-ALL initiation, driving genomic instability secondary to an increase in RS. As the pro-proliferative oncogenic program establishes and RS rises, leukemic cells are naturally selected for their ability to upregulate CHK1 as a means to maintain RS levels compatible with cell viability.

\section{CONFLICT OF INTEREST}

No potential conflicts of interest were disclosed.

João T. Barata: Instituto de Medicina Molecular, Faculdade de Medicina, Universidade de Lisboa, Lisbon, Portugal

Correspondence: João T. Barata, email joao_barata@medicina.ulisboa.pt

Keywords: CHKI, replication stress, T-ALL, T-cell acute lymphoblastic leukemia, targeted therapy

Received: July 18, 2015

Published: August 12, 2015

\section{REFERENCES}

1. Toledo LI, et al. Mol Oncol. 2011; 5: 368-373.

2. Lecona E, et al. Exp Cell Res. 2014; 329: 26-34.

3. Zhang Y, et al. Int J Cancer. 2014; 134: 1013-1023.

4. Carrassa L, et al. Cell Cycle. 2011; 10: 2121-2128.

5. Sarmento LM, et al. Oncogene. 2015; 34: 2978-2990.

6. Barata JT, et al. Blood. 2001; 98: 1524-1531.

7. De Keersmaecker K, et al. Nat Med. 2010; 16: 1321-1327. 